\title{
Forscher sprühen wider den Teufelskreis
}

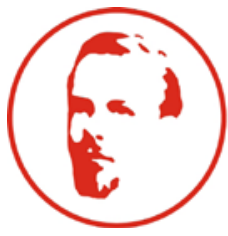

DZD

Deutsches Zentrum

für Diabetesforschung

\section{Die Entstehung von Übergewicht und Diabetes wird auch durch veränderte Hormoneinflüsse im Gehirn gefördert. Mithilfe der Forschungsarbeit von Dr. Ste- phanie Kullmann von der Uni Tübingen und ihren DZD-Kollegen soll ein entsprechender Teufelskreis durchbrochen werden.}

\section{Wie wirkt Insulin im Gehirn?}

Kullmann: Insulin wirkt auf das Essverhalten, den Stoffwechsel, das Gedächtnis sowie den Gemütszustand über Rezeptoren in bestimmten Hirnregionen. Die Rezeptoren sind besonders im Hypothalamus und kortikostriatalen Regionen verbreitet. Eine erhöhte Insulinkonzentration nach dem Essen signalisiert dem Gehirn „Ich bin satt“ und versetzt es über das Belohungssystem in Zufriedenheit, was die weitere Kalorienzufuhr verhindern soll. Wir konnten nun zeigen, dass Insulin auch Verknüpfungen zwischen den Hirnregionen verstärkt. Dies könnte erklären, warum sich Insulin im Gehirn nicht nur aufs Essverhalten auswirkt, sondern auch Gedächtnisprozesse günstig beeinflusst.

Welche Auswirkungen hat die Insulinresistenz des Gehirns? Kullmann: Von Insulinresistenz im Gehirn sprechen wir, wenn Hirnregionen unempfindlicher werden und Insulin dort nicht mehr wirkt. Mit bildgebenden Verfahren konnten wir beim Menschen zeigen, dass schon moderates Übergewicht die Insulinwirkung im Gehirn deutlich reduziert oder aufhebt. Personen mit Insulinresistenz im Gehirn haben mehr Bauchfett und weitere Risiken für Typ-2-Diabetes. Auch beim Abnehmen sind sie weniger erfolgreich. Dies liegt daran, dass der Insulineffekt im Hypothalamus dessen Wirkung im übrigen Körper beeinflusst. Dies konnten wir zeigen, indem wir Probanden Insulin per Nasenspray gaben und deren Hirnaktivität und die Insulinwirkung in Kör- pergeweben untersuchten. Des Weiteren erzeugt der reduzierte Insulineffekt im Hirn vermehrt Appetit, selbst nach ausreichender Kalorienzufuhr. Ein Teufelskreis, der schwer zu brechen ist.

Woran arbeitet das DZD, um die Rolle des Gehirns bei Diabetes noch besser zu verstehen?

Kullmann: Ob Übergewicht Folge oder Ursache der Insulinresistenz im Gehirn ist, kann noch nicht abschließend beantwortet werden. Unseren Untersuchungen zufolge bessert die einmalige Gabe von nasalem Insulin die Insulinsensitivität sowie die Glukoseaufnahme im Körper über mehrere Stunden und unterdrückt das Hungergefühl. Diese Erkenntnis ist für Personen besonders wichtig, die wenig Erfolg mit konventionellen Diät- und Sportinterventionen hatten (Non-Responder) und somit ein besonders hohes Typ-2-Diabetes-Risiko haben. Daher prüfen wir aktuell im DZD, ob eine längerfristige Gabe von nasalem Insulin bei NonRespondern seine Wirkung in Gehirn und Körper verbessern kann. Auch bei Personen mit erhöhter genetischer Disposition zu Übergewicht wollen wir die Insulinwirkung im kortikostriatalen Schaltkreis gezielt verbessern. In einem bereichsübergreifenden Forschungsprojekt, der brain academy, untersuchen DZD-Experten zudem die Rolle des Gehirns bei der Diabetesentstehung.

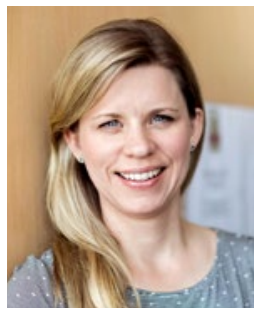

Dr. Stephanie Kullmann

Institut für Diabetesforschung und Meta-bolische Erkrankungen (IDM) des Helmholtz Zentrum München an der Eberhard-Karls-Universität Tübingen Otfried-Müller-Straße 47, 72076 Tübingen stephanie.kullmann@med.uni-tuebingen.de

Grundlagenforschung, Epidemiologie und Klinik -

Das Deutsche Zentrum für Diabetesforschung im Überblick

Im Deutschen Zentrum für Diabetesforschung (DZD) arbeiten Experten aus Grundlagenforschung, Epidemiologie und klinischer Anwendung deutschlandweit zusammen. Durch diesen translationalen Forschungsansatz können Beobachtungen aus epidemiologischen Studien im Labor überprüft und die Ergebnisse aus dem Labor schneller in die klinische Anwendung überführt werden. Ziel des DZD ist es, die Erkenntnisse der Diabetesforschung möglichst schnell zum Patienten zu bringen, um Diabetes vorzubeugen und zu behandeln sowie Folgeerkrankungen zu vermeiden.

Partner im DZD sind das Deutsche Diabetes-Zentrum in Düsseldorf, das Deutsche Institut für Ernährungsforschung in Potsdam-Rehbrücke, das Helmholtz Zentrum München - Deutsches Forschungszentrum für Gesundheit und Umwelt, das Institut für Diabetesforschung und Metabolische Erkrankungen des Helmholtz Zentrum München an der Eberhard-Karls-Universität Tübingen und das Paul-Langerhans-Institut Dresden des Helmholtz Zentrum München am Universitätsklinikum Carl Gustav Carus der Technischen Universität Dresden.

Zur Komplettierung und Stärkung der wissenschaftlichen Kompetenz integrierte das DZD einzelne Diabeteswissenschaftler und -forschungsgruppen an den Universitäten in Heidelberg, Köln, Leipzig, München und Lübeck als assoziierte Partner. Weiterhin sind Wissenschaftler des ehemaligen Kompetenznetzes Diabetes mellitus Projektpartner im DZD.

Weitere Infos: www.dzd-ev.de 